\title{
Persistent pain prevalence and medical management
}

\author{
Eldon Tunks MD FRCPC
}

$I^{2}$ $\mathrm{n}$ this issue, Moulin et al (pages 179-184) report an epidemiological survey of persistent pain and medical treatment of this pain in Canada. Two thousand twelve adult Canadians were included in a randomized sample and were interviewed by telephone. The sample was stratified to correspond to the Canadian population's prevalences of sex, age and region, according to the 1996 census data. For this study, the Canadian Ipsos-Reid Express Omnibus survey was used.

Moulin et al (pages 179-184) selected the 'chronic pain' sample according to the criteria, "pain that is continuous or intermittent, or suffering from a condition that gives you pain that is continuous or intermittent" in Canadians aged 18 to 75 years and "not related to cancer". The 2012 respondents represented a response rate of $19.1 \%$. Of these, $29 \%$ reported 'chronic pain' (27\% of men and $31 \%$ of women). The prevalence of persistent pain increased with age; in the age group 18 to 34 years, the prevalence was $22 \%$, from age 35 to 54 years the prevalence was $29 \%$ and in those aged 55 years or more the prevalence was 39\%.

On first glance, these data do not correspond with estimates from other epidemiological studies. What is the explanation? In any epidemiological survey, it is important to consider the interview methods and the questions used to define the study sample. For example, another Canadian survey of persistent pain (1) reported a $10.8 \%$ prevalence of persistent pain in the population aged 18 years or older, with an age-related increase in persistent pain. The questions used to define persistent pain in the Crook et al (1) study were "often troubled with pain" and "experienced noteworthy pain within the past two weeks". Crook et al (1) noted that these questions would identify patients with mostly continuous pain and would exclude patients who regarded their pain as intermittent. By contrast, the criteria of Moulin et al (pages 179-184) would likely include those with continuous pain as well as those with intermittent pain. Taking this into account, there is likely no contradiction between the results reported by Moulin et al (pages 179-184) and the results of earlier studies, but the samples studied are not identical.

In the second part of their study, a different sample of 340 chronic pain sufferers, drawn from the Ipsos-Reid Consumer Panel and who were taking prescription medication for pain, were interviewed in detail. The prevalence of women in this sample was $65.3 \%$. All respondents were asked to rate their pain on a numerical rating scale; the reported prevalence of mild, moderate and severe pain was $20.3 \%, 47.9 \%$ and $31.7 \%$, respectively. While there was a small difference in the reported mean pain intensity between men and women ( 6.0 mean pain intensity versus 6.4 mean pain intensity, respectively), older patients reported somewhat lower intensities of pain than did younger patients. Patients aged 18 to 34 years reported a mean pain intensity of 6.7 , respondents aged 35 to 54 years reported a mean pain intensity of 6.6 and those above age 55 years reported a mean pain intensity of 5.9. The mean duration of pain in this group of 340 patients was 10.7 years. Patients with severe pain reported a mean duration of 13.2 years, those with moderate pain reported a mean duration of 9.4 years and those with mild pain indicated a mean duration of 9.5 years.

With regard to treatment, patients using opioids reported a mean pain intensity of 7.1, compared with 6.0 for those not using opioids. The authors wondered if this might be an indication of the failure of opioid treatment. While this could be the case in some instances, it may also indicate that patients with pain that is more serious are more likely to be given opioids, or that patients given opioids are more likely to assume that their pain is greater.

It is notable that this sample of 340 resembles members of the general population more closely than it resembles the population of patients referred to pain clinics; of this group

McMaster University, Pain Management, Chedoke Rehabilitation Centre, Hamilton Health Sciences, Hamilton, Ontario

Correspondence: Dr E Tunks, McMaster University, Pain Management, Chedoke Rehabilitation Centre, Hamilton Health Sciences, PO Box 2000, Station LCD, Hamilton, Ontario L8N 325. Telephone 905-521-2100 extension 77038, fax 905-527-7927,

e-mailtunks@hhsc.ca 
$38 \%$ were employed, $22 \%$ were self employed or homemakers, $25 \%$ were retired and $7 \%$ were unemployed. They reported a mean of 9.3 absent workdays due to pain over the past year, and those with severe pain reported a mean of 16 absent workdays.

However, quality of life was impaired in the majority of those with persistent pain: $49 \%$ reported great difficulty attending social or family events; $61 \%$ reported impairment in their usual recreation; and $58 \%$ were unable to manage their usual daily activity at home. The authors emphasized that despite a prevalence of almost $30 \%$ of chronic noncancer pain, twice as many patients had received antiinflammatories than had received opioids, and major opioids had been received by only $7 \%$. Barriers to the prescription or use of opioids were that $16 \%$ found that certain analgesic medications were unaffordable; $69 \%$ believed that strong analgesics like morphine were addictive; 52\% thought that they were too strong for their pain; and 29\% thought opioids should be reserved for terminal illness. The authors interpret this to mean that opioids were not used enough to relieve pain, and they make a plea for a less restrictive attitude toward prescribing strong opioids for serious pain.

Before we accept this interpretation without criticism, we should note that the reported percentage of types of medication used adds up to about $122 \%$, indicating that many patients were using more than one type of medication. Forty-nine per cent were prescribed one or more antiinflammatory agents, while $22 \%$ reported taking an opioid analgesic. In this group of patients taking prescribed medication, $40.2 \%$ were taking conventional nonsteroidal antiinflammatory drugs, $25.9 \%$ were taking cyclooxygenase-2 inhibitors and $8.8 \%$ were taking corticosteroids. This compares with $15.2 \%$ taking codeine and $7 \%$ taking major opioids. Eighteen per cent reported the use of adjuvant analgesics: antidepressants were taken by $6.2 \%$ of patients, anticonvulsants by $5 \%$ and $7.1 \%$ were taking other medications such as muscle relaxants or antimigraine drugs. Thirty per cent reported taking over-the-counter analgesics as well as prescription medications.

Furthermore, body pain locations (back, legs, neck, head) were surveyed, but the pain mechanisms are not known. Not all pain is equal, and not all analgesics are equal. Possible pain mechanisms include mechanical, inflammatory, ischemic, neuropathic, visceral, headache and many other possibilities. These possible mechanisms often respond to quite different treatments, and do not respond equally to opioids, however 'strong' the opioids are presumed to be. For example, night pain from an osteoid osteoma will respond more to common acetylsalicylic acid than to morphine, and for some neuralgias, tricyclics or antidepressants may be more effective than opioids or anti- inflammatories. The differential responsiveness to opioids has been noted by Jadad et al (2), and the World Health Organization analgesic ladder, which is based on the notion of weak and strong analgesics, has not been demonstrably effective, partly because it does not take into account pain mechanisms for which a given agent may be more or less appropriate (3). The choice of the best analgesia depends not only on whether it is a strong opioid, but also on the match between the pain mechanism and the type of analgesic or adjuvant. In the aging population, degenerative conditions are increasingly important as causes of pain and dysfunction. For articular degenerative conditions, antiinflammatories may be appropriate and effective, and for painful neuropathies, the appropriate agencies may be anticonvulsants or tricyclics, with or without adjunctive use of opioids. However, the concerns stated by Moulin et al (pages 179-184) should not be disregarded, because the unwarranted negative attitudes documented by their survey, as well as the barrier of the prescription cost of sustained release opioids and other preparations, could be real barriers to the appropriate use of a range of analgesic options including opioids. Overcoming these barriers requires patient and professional education as well as changes to the existing drug benefit system to permit persistent pain sufferers to have easier access to the most commonly used pain relief medications.

Lest it be thought that pain relief management focuses only on drugs, the authors found that most patients were also receiving some form of nonpharmacological treatment, including exercise $(74 \%)$, relaxation therapy (43\%), physiotherapy $(28 \%)$, massage $(22 \%)$, transcutaneous electrical nerve stimulation $(12 \%)$ and acupuncture $(9 \%)$.

It is important not to equate pain relief simply with analgesics, opioids, nerve blocks or behaviour therapy. The proper assessment of the patient, identification of relevant pain mechanisms and comorbid conditions, and appropriate assessment of efficacy versus risk versus cost for possible treatment options, are all necessary as a prelude to comprehensive evidence-based pain relief treatments and strategies to manage comorbid problems. They are all essential ingredients. The paper by Moulin et al (pages 179-184) goes a long way toward supporting a rationale for better pain relief in Canada.

\section{REFERENCES}

1. Crook J, Rideout E, Browne G. The prevalence of pain complaints in a general population. Pain 1984;18:299-314.

2. Jadad AR, Carroll D, Glynn CJ, Moore RA, McQuay HJ. Morphine responsiveness of chronic pain: Double-blind randomized crossover study with patient-controlled analgesia. Lancet 1992;339:1367-71.

3. Jadad AR, Browman GP. The WHO ladder for cancer pain management: Stepping up the quality of its evaluation. JAMA 1995;274:1870-3.

The views expressed in this editorial are those of the authors and are not intended to reflect the opinions of the Canadian Pain Society or Pulsus Group Inc 


\title{
La prévalence et la prise en charge médicale de la douleur persistante
}

\author{
Eldon Tunks, MD FRCPC
}

$\mathrm{D}^{\mathrm{a}}$ ans le présent numéro, Moulin et coll. (pages 179-184) rendent compte d'une enquête épidémiologique sur la douleur persistante et le traitement de cette douleur au Canada. Deux mille douze adultes canadiens ont été inclus dans un échantillonnage aléatoire et ont été interrogés par téléphone. L'échantillonnage a été stratifié afin de correspondre à la prévalence de la population canadienne pour ce qui est du sexe, de l'âge et de la région, conformément aux données du recensement de 1996. Pour cette étude, l'enquête canadienne Ipsos-Reid Express a été utilisée.

Moulin et coll. (pages 179-184) ont sélectionné des échantillons de «douleur chronique » selon le critère « une douleur constante ou intermittente ou une maladie qui cause une douleur constante ou intermittente ", " non reliée au cancer », chez des Canadiens de 18 à 75 ans. Les 2012 répondants ont procuré un taux de réponse de 19,1\%. De ce nombre, $29 \%$ ont déclaré souffrir de « douleur chronique » (27\% d'hommes et $31 \%$ de femmes). La prévalence de la douleur persistante augmentait avec l'âge. Dans le groupe des 18 à 34 ans, elle correspondait à $22 \%$, dans celui des 35 à 54 ans, à $29 \%$, et dans celui des 55 ans et plus, à $39 \%$.

Au premier coup d'œil, ces données ne correspondent pas aux évaluations d'autres études épidémiologiques. Quelle est l'explication ? Dans toute enquête épidémiologique, il importe d'examiner les méthodes d'entrevue et les questions utilisées pour définir l'échantillonnage à l'étude. Par exemple, une autre enquête canadienne sur la douleur persistante (1) faisait état d'une prévalence de douleur persistante de 10,8 \% au sein de la population de 18 ans ou plus, ainsi que d'une augmentation de la douleur persistante reliée à l'âge. Les questions utilisées pour définir la douleur persistante dans cette étude de Crook et coll. (1) incluaient «souvent perturbé par la douleur » et « a souffert d'une douleur marquée depuis deux semaines ». Selon, Crook et coll. (1), ces questions permettaient de repérer les patients souffrant de douleurs presque constantes et d'exclure ceux qui considéraient leur douleur comme intermittente. Par contre, les critères de Moulin et coll. (pages 179-184) incluent probablement les personnes souffrant de douleurs constantes ou de douleurs intermittentes. Compte tenu de ce phénomène, il n'existe probablement pas de contradiction entre les résultats déclarés par Moulin et coll. (pages 179-184) et ceux d'études précédentes, car les échantillonnages étudiés ne sont pas identiques.

Dans la deuxième partie de l'étude, un autre échantillonnage de 340 personnes souffrant de douleurs chroniques qui prenaient des médicaments sur ordonnance contre la douleur, tiré du groupe de consommateurs Ipsos-Reid, ont passé une entrevue détaillée. La prévalence des femmes de cet échantillonnage était de 65,3\%. Tous les répondants étaient invités à évaluer leur douleur selon une échelle d'évaluation numérique. La prévalence déclarée de douleur bénigne, modérée et grave s'établissait à 20,3\%, 47,9\% et $31,7 \%$, respectivement. Même s'il existait une légère différence dans l'intensité moyenne de la douleur des hommes et des femmes (intensité moyenne de 6,0 par rapport à 6,4, respectivement), les patients plus âgés faisaient état d'une intensité de douleur un peu plus basse que les patients plus jeunes. Ainsi, les patients de 18 à 34 ans ont déclaré une intensité moyenne de la douleur de 6,7, ceux de 35 à 54 , de 6,6, et ceux de plus de 55 ans, de 5,9. La durée moyenne de la douleur au sein de ce groupe de 340 patients était de 10,7 ans. Les patients souffrant de douleur grave ont déclaré une durée moyenne de 13,2 ans, ceux souffrant de douleur modérée, une durée moyenne de 9,4 ans, et ceux souffrant de douleur légère, une durée moyenne de 9,5 ans.

Pour ce qui est du traitement, les patients utilisant des opiacés déclaraient une intensité moyenne de la douleur de 7,1 . par rapport à 6,0 pour ceux qui n'en prenaient pas. Les auteurs se sont demandés si cette réponse n'était pas une indication possible de l'échec du traitement aux opiacés. Bien que ce puisse parfois être le cas, cette réponse peut également indiquer que ceux qui souffrent des douleurs les plus graves sont plus susceptibles de recevoir des opiacés, ou que les patients qui prennent des opiacés sont plus susceptibles de considérer que leur douleur est plus grave.

Il convient de souligner que cet échantillonnage de 340 patients ressemble davantage aux membres de la population générale qu'à la population de patients orientés vers les cliniques de la douleur. Dans ce groupe, $38 \%$ occupaient un emploi, $22 \%$ étaient travailleurs autonomes ou au foyer, $25 \%$ étaient retraités et $7 \%$ étaient sans emploi. Ils ont déclaré une moyenne de 9,3 jours de travail manqués à cause de la douleur depuis un an, et ceux qui souffraient de douleurs graves ont déclaré une moyenne de 16 jours de travail manqués.

Université McMaster, Prise en charge de la douleur, Chedoke Rehabilitation Centre, Sciences de la santé de Hamilton, Hamilton (Ontario).

Correspondance: Dr E Tunks, McMaster University,Pain Management, Chedoke Rehabilitation Centre, Hamilton Health Sciences, PO Box

2000, Station LDC, Hamilton (Ontario) L8N 325. Téléphone :905-521-2100, poste 77038, télécopieur : 905-527-7927, courriel : tunks@hhsc.ca 
Cependant, la qualité de vie était perturbée pour la majorité des personnes souffrant d'une douleur persistante : $49 \%$ ont déclaré éprouver d'énormes difficultés à assister à des activités sociales ou familiales, $61 \%$ ont fait état de perturbations à leurs activités récréatives habituelles et $58 \%$ étaient incapables de gérer leurs activités quotidiennes habituelles à la maison. Les auteurs ont souligné que malgré la prévalence d'au moins $30 \%$ des douleurs non cancéreuses chroniques, deux fois plus de patients avaient reçu des anti-inflammatoires que des opiacés, et seuls $7 \%$ avaient reçu des opiacés majeurs. Les obstacles à la prescription ou à l'usage d'opiacés étaient que $16 \%$ trouvaient certains analgésiques inabordables, $69 \%$ pensaient que des analgésiques puissants comme la morphine créaient une accoutumance, $52 \%$, que les opiacés étaient trop puissants pour leur douleur et $29 \%$, qu'ils devraient être réservés aux maladies en phase terminale. Les auteurs ont interprété que les opiacés n'étaient pas assez utilisés pour soulager la douleur et ont fait un plaidoyer en faveur d'une attitude moins restrictive face à la prescription d'opiacés puissants en cas de douleur grave.

Avant d'accepter cette interprétation sans critique, nous devons souligner que le pourcentage déclaré des types de médicaments utilisés totalisait environ $122 \%$, ce qui indique que de nombreux patients prenaient plus d'un type de médicament. On avait prescrit plus d'un anti-inflammatoire à $49 \%$ des patients, tandis que $22 \%$ prenaient des analgésiques opioïdes. Dans ce groupe de patients prenant des médicaments sur ordonnance, 40,2 \% prenaient des anti-inflammatoires non stéroïdiens conventionnels, 25,9\%, des inhibiteurs de la cyclooxygénase- 2 et $8,8 \%$, des corticoïdes. Ces pourcentages se comparent à 15,2\% prenant de la codéine et $7 \%$, des opiacés majeurs. Dix-huit pour cent ont déclaré prendre des adjuvants, $6,2 \%$, des antidépresseurs, $5 \%$, des anticonvulsivants et $7,1 \%$, d'autres médicaments comme des myorelaxants ou des antimigraineux. Trente pour cent ont déclaré prendre des analgésiques en vente libre en plus de médicaments sur ordonnance.

Par ailleurs, le foyer de la douleur (dos, jambes, cou, tête) a été sondé, mais les mécanismes de la douleur demeurent inconnus. Aucune douleur n'est équivalente, et les analgésiques ne sont pas tous équivalents. Parmi les mécanismes possibles de la douleur, soulignons les douleurs mécanique, inflammatoire, ischémique, névropathique, viscérale, migraineuse et de nombreuses autres possibilités. Ces mécanismes possibles réagissent souvent à des traitements très différents, et ne réagissent pas tous de la même façon aux opiacés, quelle qu'en soit la puissance présumée. Par exemple, les douleurs nocturnes d'un ostéome ostéoïde réagiront mieux à l'acide acétylsalicylique courant qu'à la morphine, et dans le cas de certaines névralgies, les tricycliques ou les antidépresseurs peuvent se révéler plus efficaces que les opiacés ou les anti-inflammatoires. La réaction différentielle aux opiacés a été soulignée par Jada et coll. (2), et l'ef- ficacité de l'échelle analgésique de l'OMS, fondée sur la notion d'analgésiques faibles ou puissants, n'est pas probante, en partie parce qu'elle ne tient pas compte des mécanismes de la douleur pour lesquels un agent donné peut convenir plus ou moins (3). Le choix du meilleur analgésique dépend non seulement de la puissance de l'opiacé, mais également de la correspondance entre le mécanisme de la douleur et le type d'analgésique ou d'adjuvant. Au sein de la population vieillissante, les maladies dégénératives constituent de plus en plus des causes de douleur ou de dysfonction. En cas de troubles dégénératifs articulaires, les anti-inflammatoires peuvent être convenables et efficaces et, dans le cas de névropathies douloureuses, les tricycliques ou les anticonvulsivants peuvent convenir mieux, avec ou sans l'ajout d'opiacés. Cependant, les préoccupations soulignées par Moulin et coll. (pages 179-184) ne devraient pas être négligées, car les attitudes négatives non fondées et le coût des ordonnances d'opiacés à libération prolongée ou d'autres préparations peuvent constituer de véritables obstacles à l'usage convenable d'une gamme d'analgésiques, y compris les opiacés. Pour franchir ces obstacles, il faut éduquer à la fois les patients et les professionnels et modifier le système d'assurance-maladie actuel pour permettre aux personnes qui souffrent de douleurs constantes de profiter d'un meilleur accès aux analgésiques les plus courants.

Il ne faut toutefois pas penser que la prise en charge de la douleur n'est axée que sur les médicaments, car les auteurs ont découvert que la plupart des patients bénéficiaient d'une autre forme de traitement non pharmacologique, y compris l'exercice (74\%), la thérapie de relaxation (43\%), la physiothérapie (28\%), les massages (22\%), la stimulation nerveuse électrique transcutanée (12\%) et l'acupuncture $(9 \%)$.

Il est important de ne pas faire correspondre le soulagement de la douleur seulement avec les analgésiques, les opiacés, le blocage nerveux ou la thérapie behaviorale. Une bonne évaluation du patient, le dépistage des méca-nismes pertinents de la douleur et des troubles comorbides, de même qu'une évaluation convenable de l'efficacité par rapport au risque et au coût des possibilités de traitement sont tous des éléments nécessaires pour commencer à comprendre des stratégies et traitements probants visant à soulager la douleur, afin de prendre en charge les troubles comorbides. Ce sont tous des ingrédients essentiels. Cet article de Moulin et coll. (pages 179-184) devrait réussir à justifier l'adoption d'un meilleur soulagement de la douleur au Canada.

\section{RÉFÉRENCES}

1. Crook J, Rideout E, Browne G. The prevalence of pain complaints in a general population. Pain 1984;18:299-314.

2. Jadad AR, Carroll D, Glynn CJ, Moore RA, McQuay HJ. Morphine responsiveness of chronic pain: Double-blind randomized crossover study with patient-controlled analgesia. Lancet 1992;339:1367-71.

3. Jadad AR, Browman GP. The WHO ladder for cancer pain management: Stepping up the quality of its evaluation. JAMA 1995;274:1870-3.

Les opinions ici exprimées n'engagent que les auteurs et ne reflètent pas nécessairement celles de la Société canadienne pour le traitement de la douleur ou de Pulsus Group Inc 


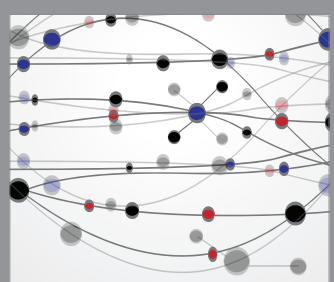

The Scientific World Journal
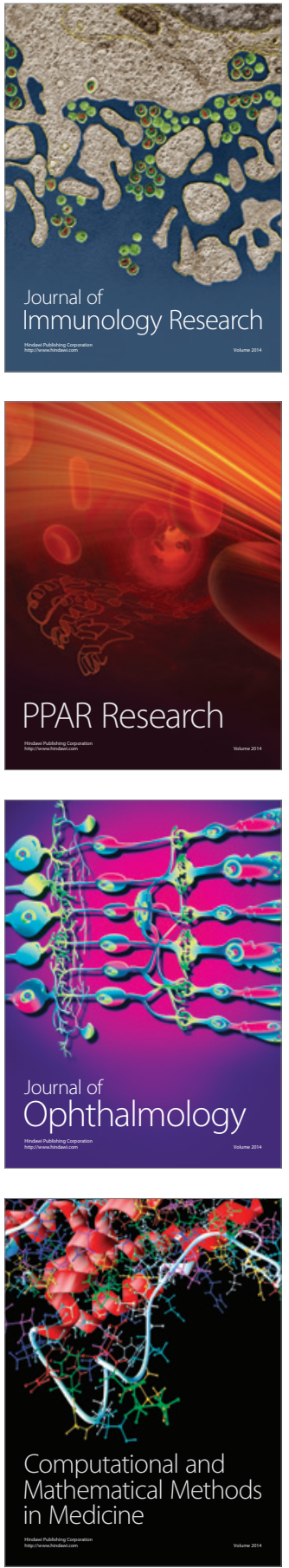

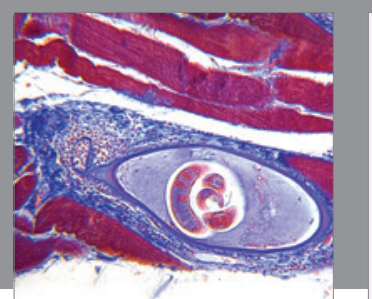

Gastroenterology Research and Practice

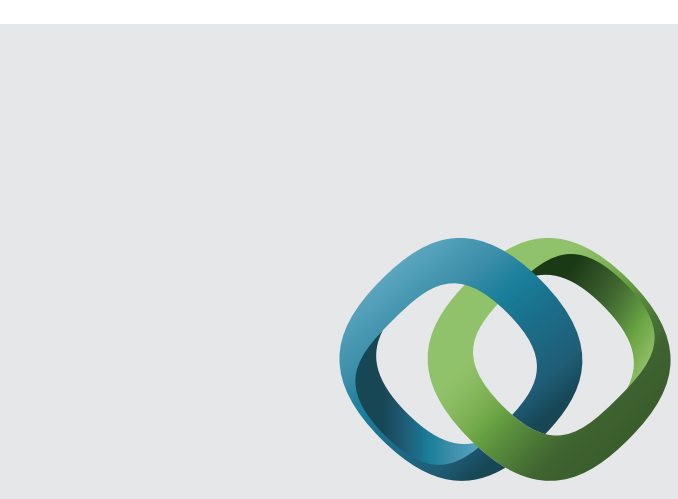

\section{Hindawi}

Submit your manuscripts at

http://www.hindawi.com
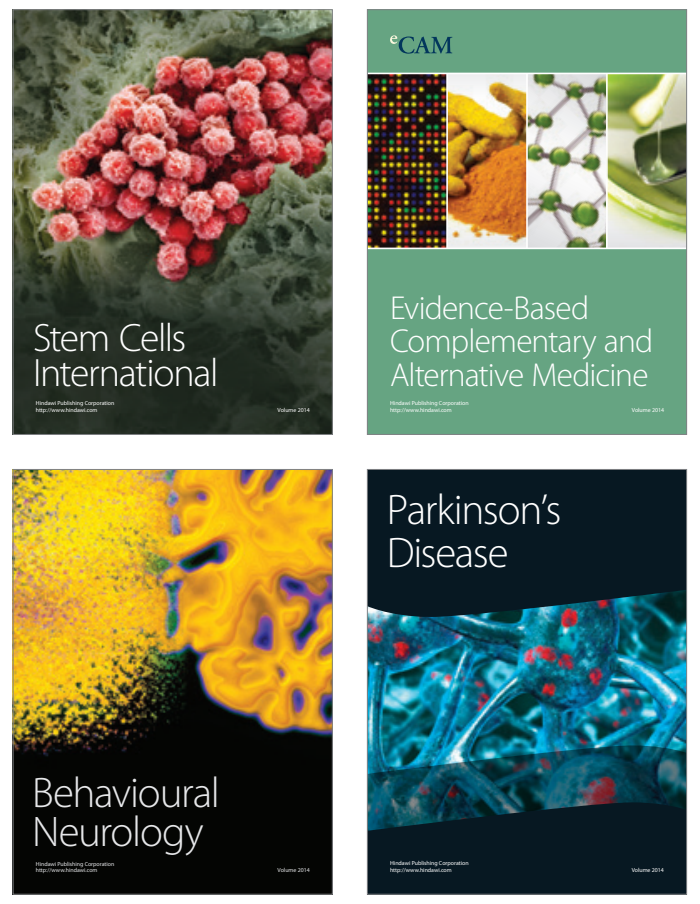
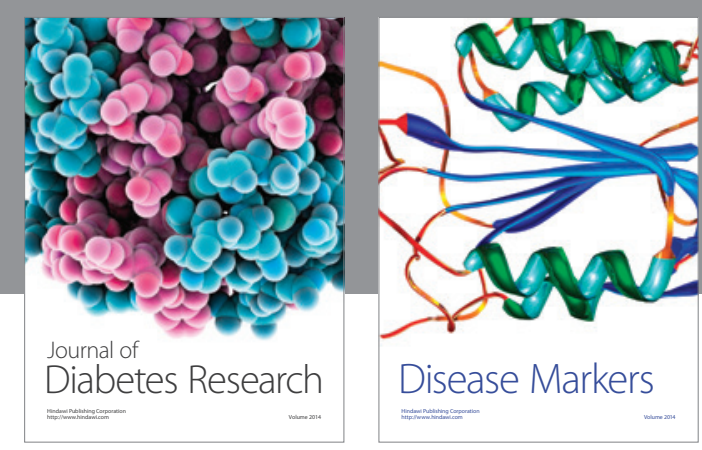

Disease Markers
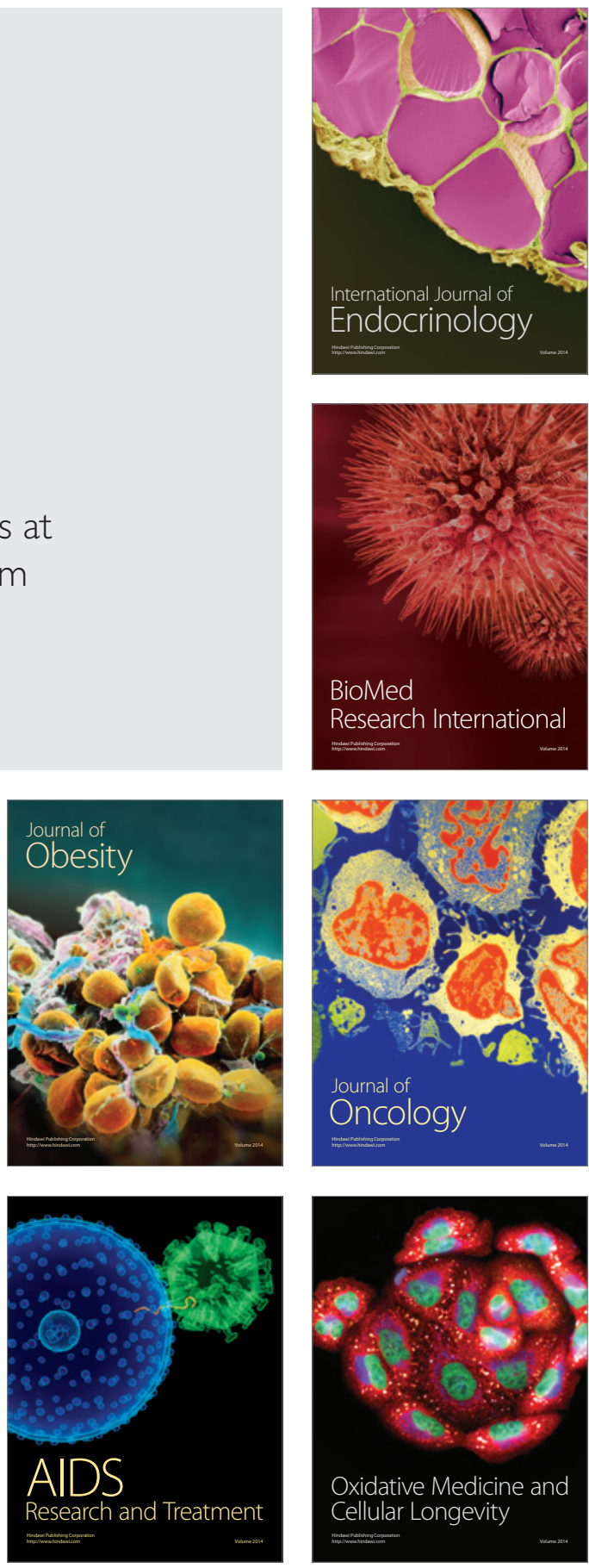